\title{
Predictors of visual functional outcome following treatment for cavernous sinus meningioma
}

\author{
Nida Fatima, MD, ${ }^{1}$ Victoria Y. Ding, MS, ${ }^{2}$ Summer S. Han, PhD, ${ }^{2}$ Steven D. Chang, MD, ${ }^{1}$ and \\ Antonio Meola, MD, PhD'
}

${ }^{1}$ Department of Neurosurgery, and ${ }^{2}$ Quantitative Sciences Unit, Stanford University School of Medicine, Stanford, California

OBJECTIVE Cavernous sinus meningioma (CSM) can affect visual function and require expeditious treatment to prevent permanent visual loss. Authors of this retrospective study sought to determine the factors associated with visual functional outcomes in CSM patients treated with surgery, stereotactic radiosurgery (SRS), or stereotactic radiation therapy (SRT), alone or in combination.

METHODS Consecutive patients with CSM who had presented at an academic tertiary care hospital from 2000 to 2018 were identified through retrospective chart review. Visual function-visual eye deficit (VED), optic disc (OD) appearance, intraocular pressure (IOP), and extraocular movement (EOM) - was assessed before and after treatment for CSM. VED with visual acuity (VA) $\leq 20 / 200$ and visual field defect $\geq-11 \mathrm{~dB}$, pale OD appearance in the ipsilateral or contralateral eye, increased ipsilateral IOP, and/or EOM restriction were defined as a poor visual functional outcome. Multivariable logistic regression was used to evaluate the associations between pretreatment visual functional assessment and posttreatment visual outcomes.

RESULTS The study cohort included 44 patients (73\% female; median age 55 years), with a median clinical follow-up of 14 months. Ipsilateral VED improved, remained stable, or worsened, respectively, in $0 \%, 33.4 \%$, and $66.6 \%$ of the patients after subtotal resection (STR) alone; in 52.6\%, 31.6\%, and $15.8 \%$ after STR plus radiation treatment; in $28.5 \%$, $43.0 \%$, and $28.5 \%$ after gross-total resection (GTR) alone; and in $56.3 \%, 43.7 \%$, and $0 \%$ after radiation treatment (SRS or SRT) alone. Contralateral VED remained intact in all the patients after STR alone and those with radiation treatment (SRS or SRT) alone; however, it improved, remained stable, or worsened in $10.5 \%, 84.2 \%$, and $5.3 \%$ after STR plus radiation treatment and in $43.0 \%, 28.5 \%$, and $28.5 \%$ after GTR alone. EOM remained intact, fully recovered, remained stable, and worsened, respectively, in $0 \%, 50 \%, 50 \%$, and $0 \%$ of the patients after STR alone; in $36.8 \%, 47.4 \%, 15.8 \%$, and $0 \%$ of the patients after STR with radiation treatment; in $57.1 \%, 0 \%, 28.6 \%$, and $14.3 \%$ of the patients after GTR alone; and in $56.2 \%, 37.5 \%, 6.3 \%$, and $0 \%$ of the patients after radiation treatment (SRS or SRT) alone.

In multivariable analyses adjusted for age, tumor volume, and treatment modality, initial ipsilateral poor VED (OR 10.1, $95 \% \mathrm{Cl} 1.05-97.2, p=0.04)$ and initial ipsilateral pale OD appearance (OR 21.1, 95\% Cl 1.6-270.5, $p=0.02)$ were associated with poor ipsilateral VED posttreatment. Similarly, an initial pale OD appearance (OR 15.7, 95\% Cl 1.3-199.0, $p=0.03$ ), initial poor VED (OR 21.7, 95\% CI 1.2-398.6, $p=0.03$ ), and a higher IOP in the ipsilateral eye (OR 55.3, 95\% $\mathrm{Cl} 1.7-173.9, p=0.02$ ) were associated with an ipsilateral pale OD appearance posttreatment. Furthermore, a higher initial ipsilateral IOP (OR 35.9, 95\% CI 3.3-400.5, $p=0.004$ ) was indicative of a higher IOP in the ipsilateral eye posttreatment. Finally, initial restricted EOM was indicative of restricted EOM posttreatment (OR 20.6, 95\% CI 18.7-77.0, p = 0.02).

CONCLUSIONS Pretreatment visual functional assessment predicts visual outcomes in patients with CSM and can be used to identify patients at greater risk for vision loss.

https://thejns.org/doi/abs/10.3171/2020.2.JNS193009

KEYWORDS cavernous sinus meningioma; visual eye deficit; stereotactic radiosurgery; stereotactic radiation therapy; oncology

\footnotetext{
ABBREVIATIONS CSM = cavernous sinus meningioma; EBRT = external beam radiation therapy; EOM = extraocular movement; GTR = gross-total resection; IMRT = intensity-modulated radiation therapy; IOP = intraocular pressure; NLP = no light perception; OD = optic disc; $S R S=$ stereotactic radiosurgery; SRT = stereotactic radiation therapy; STR = subtotal resection; VA = visual acuity; VF = visual field; VED = visual eye deficit.
}

SUBMITTED November 5, 2019. ACCEPTED February 28, 2020.

INCLUDE WHEN CITING Published online May 15, 2020; DOI: 10.3171/2020.2.JNS193009. 
$\mathrm{D}$ ESPITE the advancement of operative tools and techniques in navigating anatomical terrain, $, 1,2$ cavernous sinus meningioma (CSM) still poses formidable neurosurgical challenges. Located close to critical neurovascular structures, these slow-growing, benign lesions may infiltrate this anatomy ${ }^{2-4}$ and in turn hamper chances for complete resection., ${ }^{2,5}$ Furthermore, resection of these tumors has been reported to cause permanent cranial neuropathy in $6 \%-42 \%$ of cases, ${ }^{6,7}$ with a mortality rate up to $10 \%{ }^{6,7}$ Therefore, alternative treatment modalities in the form of stereotactic radiosurgery (SRS) and stereotactic radiotherapy (SRT) - either as primary treatment or as an adjunct to surgery-are associated with better functional outcomes and tumor control in $\mathrm{CSM}^{8,9}$ In terms of the preservation of visual function in CSM, previous studies have suggested that surgery offers little to no benefit ${ }^{10,11}$ however, radiation therapy (SRS or SRT) leads to maximal conservation and/or improvement of vision in affected patients. ${ }^{12,13}$

No previous studies have investigated predictive factors for visual functional outcome in CSM. In this study, we aimed to explore the predictive value of various pre- and peritreatment factors, including demographics, tumor characteristics, treatment modalities, and initial visual assessment, for posttreatment visual functional outcomes in CSM. Understanding these factors is paramount in choosing the best course of treatment for the patient.

\section{Methods \\ Study Population and Treatment}

Retrospective chart review of a prospectively maintained electronic database identified consecutive patients with CSM treated between 2000 and 2018. We included patients with meningiomas arising from the cavernous sinus and causing visual functional impairment. We excluded patients with optic nerve sheath or orbital apex meningioma, as well as those with incomplete data on visual assessment. This study was approved by the Stanford Hospital Institutional Review Board; however, patient consent was not required as it was a retrospective study with no identifiable patient data.

All cases were operated on by specialized neurosurgeons at our institution, with surgical approaches dependent upon tumor location and clinical judgment.

\section{Ophthalmological Examinations}

\section{Visual Eye, Optic Disc, and IOP Assessment}

The visual functions of visual acuity (VA) and visual field (VF) were assessed before and after surgery. VA was determined using the Snellen chart with correction, while the Humphrey visual field tool (Humphrey Visual Field Analyzer 5.1 for Series II instruments) was used for perimetric assessment. VF deficits were categorized into three grades ${ }^{14}$ by taking into account the mean corrected defect (mean deviation as per the patient age): grade $1=-1.8$ to $-4 \mathrm{~dB}$, grade $2=-4.1$ to $-11 \mathrm{~dB}$, and grade $3=-11 \mathrm{~dB}$ or greater. Visual eye deficit (VED) was defined as follows: none, VA $\geq 20 / 32$ and normal VF; mild, VA $\geq 20 / 32$ and grade 1 VF defect; moderate, VA 20/32-20/200 and grade
2 VF defect; severe, VA $\leq 20 / 200$ and/or grade 3 VF defect; and no light perception (NLP).

Fundoscopy was performed to assess the optic discs (ODs) for pallor and swelling, and the intraocular pressure (IOP) was measured using tonometry.

\section{Extraocular Movement Assessment}

The ocular motility test was used to assess the movement of extraocular muscles of the eye. On assessment, if there was limited mobility of gaze in any direction, it was termed "restricted," whereas "full" was defined as equal and unlimited movement of the eye in all gaze directions.

\section{Timing of the Examination and Follow-Up}

The ophthalmological examination was performed by an ophthalmologist before and after surgery and/or radiation therapy. Per our institutional protocol, all patients were assessed immediately posttreatment to identify any visual changes; however, we only considered data from visual assessments performed at the last follow-up. Patients usually underwent clinical and radiological follow-up at 6 months, 1 year, and 1.5 years after treatment.

\section{Statistical Analysis}

Demographic and clinical characteristics are summarized as counts and percentages for categorical characteristics and as medians with ranges for continuous characteristics. Logistic regression analysis was performed to determine the effect of various pre- and peritreatment visual parameters, as well as potential confounders (e.g., age, primary treatment modality, and tumor volume), on posttreatment outcome measures, which included ipsilateral VED, OD appearance, IOP, and extraocular movement (EOM). The outcomes were further categorized as poor VED (severe VED to NLP) versus good VED (no/mild/ moderate VED), poor OD appearance (ipsilateral and/or contralateral pallor) versus good OD appearance (normal OD), poor IOP (ipsilateral high IOP) versus good IOP (normal ipsilateral IOP), and full versus restricted EOM. Our classification of good or poor VED was based on blindness as defined by the American Medical Association $^{15}$ and the Social Security Act of 1935. Accordingly, an individual is considered blind with a central visual acuity of 20/200 or less in the better eye with corrective glasses or if the peripheral field is contracted to no more than $20^{\circ}$ in the better eye. Therefore, moderate VED (VA 20/32$20 / 200$ and VF defect -4.1 to $-11 \mathrm{~dB}$ ) is part of the good outcome (no blindness), whereas severe VED and NLP belong to poor outcome (blindness). Moreover, we defined VED improvement as a 1-grade improvement in the VED in the posttreatment period compared to pretreatment status. For each outcome, parameters significant at the 0.1 alpha level on univariable analysis were then entered into a multivariable regression model. Statistical significance was assessed at the 0.05 alpha level.

We conducted subgroup analysis stratified by treatment modality to evaluate the impact on posttreatment VED and EOM. In a subgroup analysis, we compared the influence of treatment modalities on posttreatment visual functional outcomes using the chi-square test, with statistical signif- 
icance assessed at the 0.05 alpha level. Among patients treated with surgery or radiation, we also considered their surgical approach (open surgery vs endoscopic), completeness of resection (subtotal resection [STR] vs gross-total resection [GTR]), and radiation treatment modality (SRS vs SRT). IBM SPSS Statistics software version 23.0 (IBM Corp.) was used to perform all analyses.

\section{Results}

The study cohort included 44 patients ( $73 \%$ female) with a median age of 55 years (range 13-83 years) and median follow-up of 14 months (range 4-24 months). Nearly half of the patients presented with diplopia (45.4\%), followed by headache (31.8\%), dizziness (31.8\%), visual impairment (25\%), trigeminal neuralgia (4.5\%), and severe orbital pain $(4.5 \%)$. These and additional treatment details are listed in Supplemental Tables 1 and 2 and summarized in Table 1. Cohort characteristics are graphically illustrated in Fig. 1.

All CSMs treated surgically had a histopathological diagnosis of WHO grade I, except 1 tumor (case 3) that was WHO grade II and was treated successfully with no recurrence. Resection was adopted in the majority of patients (61.4\%), with GTR attained in $29.6 \%$ and STR in $70.3 \%$ of these patients. Over half of the meningiomas were on the right side $(56.8 \%)$, and the median tumor volume was 15 $\mathrm{cm}^{3}$ (range $1.4-66 \mathrm{~cm}^{3}$ ) at time of treatment.

The frontotemporal craniotomy approach was adopted in $34.1 \%$ of patients $(n=15)$, followed by the transzygomatic approach in $11.4 \%(\mathrm{n}=5)$, endonasal transsphenoidal approach in $6.8 \%(\mathrm{n}=3)$, transcranial orbitotomy in $4.5 \%(\mathrm{n}=2)$, endoscopic optic nerve decompression in $2.3 \%(\mathrm{n}=1)$, and retrosigmoid approach in $2.5 \%(\mathrm{n}=1)$. In this series, the transcranial and endoscopic approaches to the orbit were needed to resect the orbital extension of the CSM and relieve pressure on the optic nerve and/or on the cranial nerves coordinating the EOMs. In a similar fashion, the retrosigmoid approach was needed to resect a CSM with significant extension in the posterior fossa and compression to the brainstem. Almost all patients (89.5\%) with STR received adjuvant radiation therapy in the form of SRS or SRT. The median dose of SRS as second-tier or adjuvant therapy was 24 Gy (range 16-32 Gy) over a median of 3 fractions (range 3-5), whereas the median dose of SRT as adjuvant therapy was 54 Gy (range 50-63 Gy) over a median of 30 fractions (range 25-30).

SRS in the form of CyberKnife surgery (Accuray Inc.) was used as a primary treatment modality in 12 patients (27.3\%), with a median dose of 24 Gy (range 14-60 Gy) over a median of 3 fractions (range 1-5). SRT was used as a first-line treatment in 5 patients (11.4\%), with intensitymodulated radiation therapy (IMRT) in 4 patients $(9.1 \%)$ and external beam radiation therapy (EBRT) in 1 patient (2.3\%). The median dose for SRT as a first-tier therapy in CSM was 54 Gy (range 45-54 Gy) over a median of 27.5 fractions (range 25-30).

The severity of visual compromise before and after surgery is summarized in Table 2 and Figs. 2-6. Estimated odds ratios with $95 \%$ confidence intervals from univariable and multivariable logistic regression models are presented in Table 3.

\section{Pre- and Posttreatment VED \\ Ipsilateral Eye}

At the last clinical follow-up, there was improvement in VED in 20 patients (45.5\%), a worsening of visual symptoms in 6 patients (13.6\%), and no change in visual function in the remaining 18 patients $(40.9 \%)$. Ipsilateral VED improved, remained stable, or worsened, respectively, in $0 \%, 33.4 \%$, and $66.6 \%$ of the patients after STR alone; in $52.6 \%, 31.6 \%$, and $15.8 \%$ after STR plus radiation treatment; in $28.5 \%, 43.0 \%$, and $28.5 \%$ after GTR alone; and in $56.3 \%, 43.7 \%$, and $0 \%$ after radiation treatment (SRS or SRT) alone.

On univariable analysis, age, initial VED, and initial OD appearance were significantly associated with final ipsilateral poor VED. Multivariable regression analysis revealed that an initial poor VED (OR 10.1, 95\% CI 1.0597.2, $\mathrm{p}=0.04)$ and initial pale OD appearance (OR 21.1, 95\% CI 1.6-270.5, $\mathrm{p}=0.02$ ) were significant independent predictors of ipsilateral poor VED posttreatment.

\section{Contralateral Eye}

Posttreatment, there was improvement in the visual functional status in 6 patients $(13.6 \%)$, while 3 patients $(6.8 \%)$ had deterioration in their visual functional status. Contralateral VED remained intact in all the patients after STR alone and those with radiation treatment (SRS or SRT) alone; however, it improved, remained stable, or worsened in $10.5 \%, 84.2 \%$, and $5.3 \%$ after STR plus radiation treatment and in $43.0 \%, 28.5 \%$, and $28.5 \%$ after GTR alone.

Multivariable regression analysis was not performed, as no characteristics reached statistical significance in the univariable analysis.

\section{Optic Disc Appearance}

Posttreatment, 10 patients (22.7\%) had improvement in their OD appearance, while 3 patients $(6.8 \%)$ had worsening of their OD appearance.

In the univariable regression analysis, we found that age, initial VED, initial OD appearance, and initial IOP were significantly associated with the final ipsilateral pale OD appearance. Multivariable regression analysis showed that initial pale OD appearance (OR 15.7, 95\% CI 1.3-199.0, $\mathrm{p}=0.03$ ), initial poor ipsilateral VED (OR 21.7, 95\% CI 1.2-398.6, $\mathrm{p}=0.03$ ), and initial IOP (ipsilateral > contralateral; OR 55.3, 95\% CI 1.7-173.9, $\mathrm{p}=0.02$ ) were significant independent predictors of a final pale OD appearance posttreatment.

\section{Intraocular Pressure}

Pretreatment, IOP was comparable in the affected and unaffected eyes in 10 patients (22.7\%), and ipsilateral IOP was higher than contralateral IOP in 30 patients $(68.2 \%)$. Posttreatment, 15 patients (34.1\%) had equal IOP in both eyes, and 27 patients (61.4\%) had higher IOP in the ipsilateral eye than in the contralateral eye.

Regression analysis revealed that a higher initial IOP in the ipsilateral eye had greater odds of occurring posttreatment (OR 35.9, 95\% CI 3.3-400.5, p = 0.004), whereas an age $>55$ years was associated with lower odds (OR 0.11, $95 \%$ CI $0.01-0.97, \mathrm{p}=0.04$ ) of such an occurrence. 
TABLE 1. Summary of characteristics among a cohort treated for CSM

\begin{tabular}{|c|c|}
\hline Variable & No. $(\%)$ \\
\hline \multicolumn{2}{|l|}{ Age in yrs } \\
\hline Median & 54.5 \\
\hline Range & $13-83$ \\
\hline \multicolumn{2}{|l|}{ Sex } \\
\hline Male & $12(27.2)$ \\
\hline Female & $32(72.8)$ \\
\hline \multicolumn{2}{|l|}{ Presenting symptoms } \\
\hline Visual impairment & $11(25)$ \\
\hline Ipsilat eye & $11(25)$ \\
\hline Contralat eye & $0(0)$ \\
\hline Ipsilat \& contralat eye & $6(13.6)$ \\
\hline Headache & $14(31.8)$ \\
\hline Diplopia & $20(45.4)$ \\
\hline Dizziness & $14(31.8)$ \\
\hline Other* & $4(9.1)$ \\
\hline \multicolumn{2}{|l|}{ Site of CSM } \\
\hline Rt & $25(56.8)$ \\
\hline $\mathrm{Lt}$ & $19(43.2)$ \\
\hline \multicolumn{2}{|l|}{ Tumor vol in $\mathrm{cm}^{3}$} \\
\hline Median & 15 \\
\hline Range & $1.4-66$ \\
\hline \multicolumn{2}{|l|}{ Primary treatment } \\
\hline Surgery & $27(61.4)$ \\
\hline SRS & $12(27.3)$ \\
\hline SRT & $5(11.4)$ \\
\hline \multicolumn{2}{|l|}{ Primary treatment modality } \\
\hline \multicolumn{2}{|l|}{ Surgical approach } \\
\hline FTC & $15(34.1)$ \\
\hline $\mathrm{TZ}$ & $5(11.4)$ \\
\hline TCO & $2(4.5)$ \\
\hline ENTS & $3(6.8)$ \\
\hline EOND & $1(2.3)$ \\
\hline RS & $1(2.3)$ \\
\hline \multicolumn{2}{|l|}{ SRS } \\
\hline CyberKnife & $12(27.3)$ \\
\hline \multicolumn{2}{|l|}{ SRT } \\
\hline IMRT & $4(9.1)$ \\
\hline EBRT & $1(2.3)$ \\
\hline \multicolumn{2}{|l|}{ Order of treatment sequence } \\
\hline Primary surgery followed by SRS or SRT & $27(61.4)$ \\
\hline Primary SRS or SRT followed by surgery & $17(38.6)$ \\
\hline \multicolumn{2}{|l|}{ Completeness of resection } \\
\hline GTR & $8(29.6)$ \\
\hline STR & $19(70.3)$ \\
\hline \multicolumn{2}{|l|}{ Marginal prescribed doses } \\
\hline \multicolumn{2}{|l|}{ Primary treatment } \\
\hline \multicolumn{2}{|l|}{ SRS } \\
\hline Median & 24 \\
\hline Range & $14-60$ \\
\hline \multicolumn{2}{|l|}{ SRT } \\
\hline Median & 54 \\
\hline Range & $45-54$ \\
\hline
\end{tabular}

CONTINUED IN NEXT COLUMN »
» CONTINUED FROM PREVIOUS COLUMN

TABLE 1. Summary of characteristics among a cohort treated for CSM

\begin{tabular}{|c|c|}
\hline Variable & No. $(\%)$ \\
\hline \multicolumn{2}{|l|}{ Marginal prescribed doses (continued) } \\
\hline \multicolumn{2}{|l|}{ Secondary treatment } \\
\hline \multicolumn{2}{|l|}{ SRS } \\
\hline Median & 24 \\
\hline Range & $16-32$ \\
\hline \multicolumn{2}{|l|}{ SRT } \\
\hline Median & 54 \\
\hline Range & $50-63$ \\
\hline \multicolumn{2}{|l|}{ Fractions } \\
\hline \multicolumn{2}{|l|}{ Primary treatment } \\
\hline \multicolumn{2}{|l|}{ SRS } \\
\hline Median & 3 \\
\hline Range & $1-5$ \\
\hline \multicolumn{2}{|l|}{ SRT } \\
\hline Median & 27.5 \\
\hline Range & $25-30$ \\
\hline \multicolumn{2}{|l|}{ Secondary treatment } \\
\hline \multicolumn{2}{|l|}{ SRS } \\
\hline Median & 3 \\
\hline Range & $3-5$ \\
\hline \multicolumn{2}{|l|}{ SRT } \\
\hline Median & 30 \\
\hline Range & $25-30$ \\
\hline \multicolumn{2}{|l|}{ Clinical FU in mos } \\
\hline Median & 14 \\
\hline Range & $4-24$ \\
\hline Recurrence & $3(6.8)$ \\
\hline Complications & $6(13.6)$ \\
\hline Orbital pain & $2(4.5)$ \\
\hline Ophthalmoplegia & $2(4.5)$ \\
\hline Subacute infarct \& homonymous hemianopia & $1(2.3)$ \\
\hline Radiation-induced skin necrosis & $1(2.3)$ \\
\hline
\end{tabular}

ENTS = endonasal transsphenoidal surgery; EOND = endoscopic optic nerve decompression; FTC = frontotemporal craniotomy; FU = follow-up; RS = retrosigmoid approach; TCO = transcranial orbitotomy; TZ = transzygomatic approach.

* Two patients with trigeminal neuralgia and 2 with severe orbital pain.

\section{Final EOM}

Preoperatively, there was decreased motility of the ipsilateral eye in $52.3 \%$ of the patients $(n=23)$, and full movement in all gazes in $47.7 \%$ of the patients $(n=21)$. Among the 21 patients with intact EOM pretreatment, $20(95.2 \%)$ retained full EOM posttreatment. Only 1 patient with normal EOM pretreatment had restricted EOM after GTR. Among the 23 patients with restricted EOM pretreatment, full recovery was achieved in 11 patients (47.8\%) and deficit stability was maintained in 7 patients (30.4\%) after treatment; deficit worsening after treatment was observed in 5 patients (21.7\%). Among this last group of worsened patients, the worsening was attributable to a new cranial nerve deficit in 2 patients $(8.7 \%)$ and to a worsening preexisting deficit in 3 patients (13\%). In an analysis according to treatment modality, EOM remained 


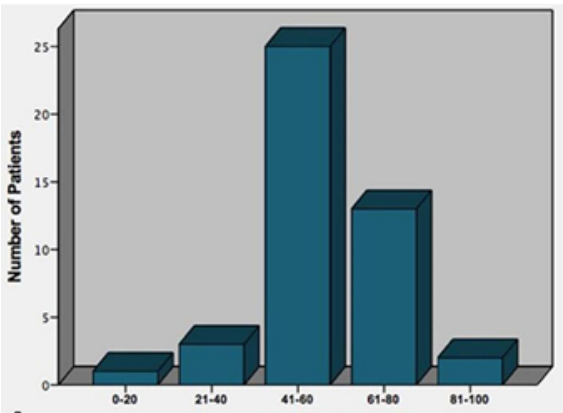

A

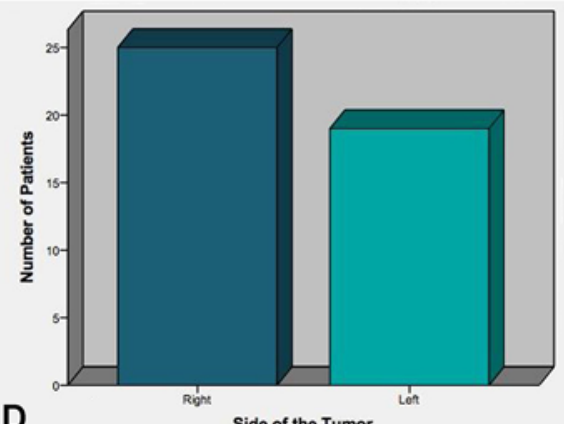

D
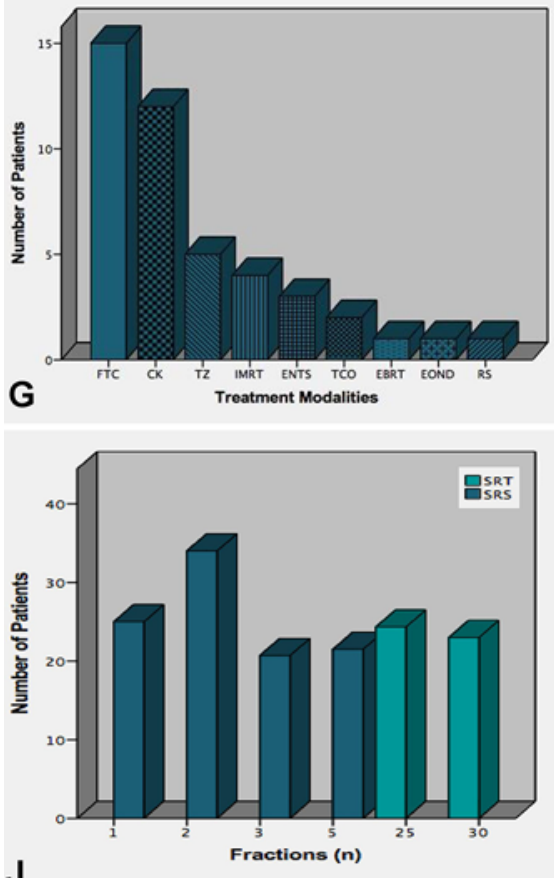

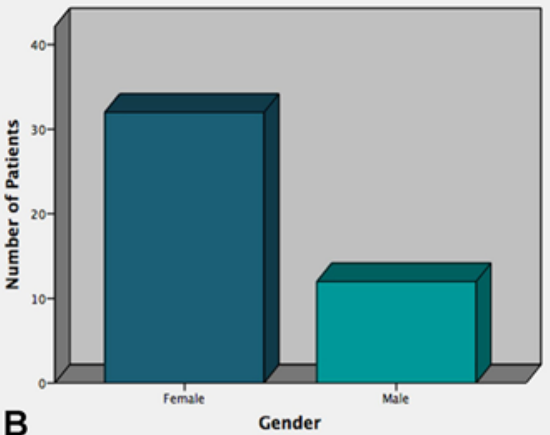

$\mathbf{B}$

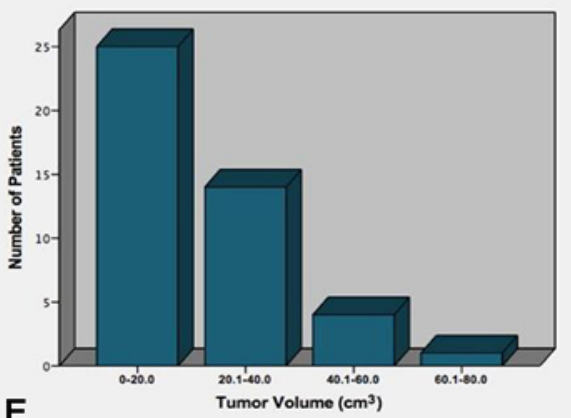

E
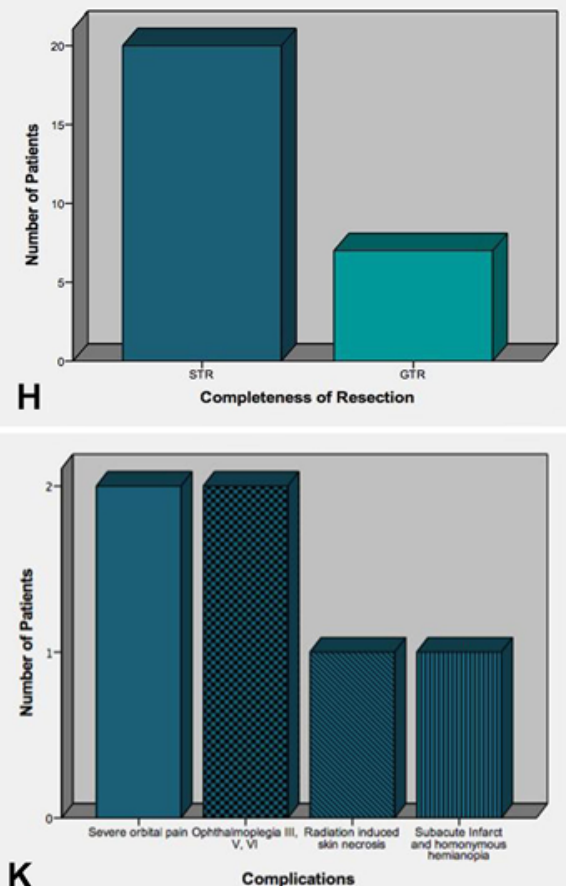

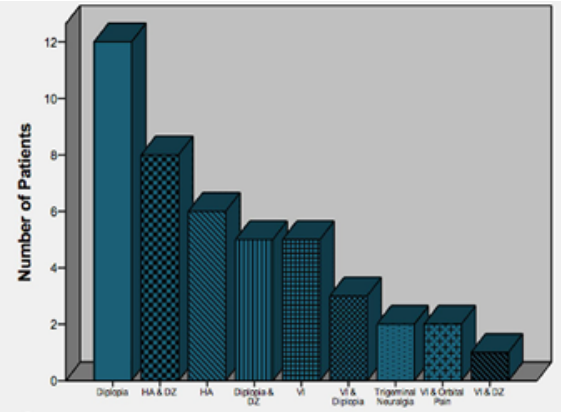

c

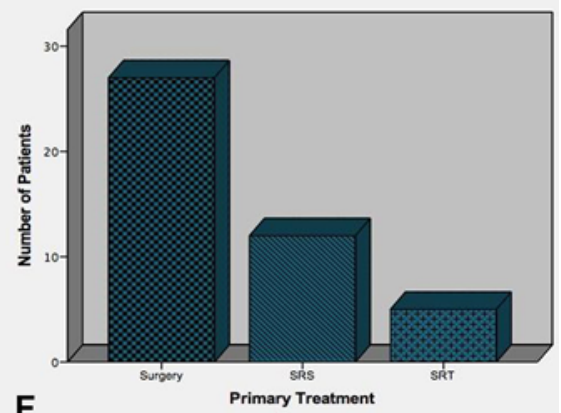

$\mathbf{F}$
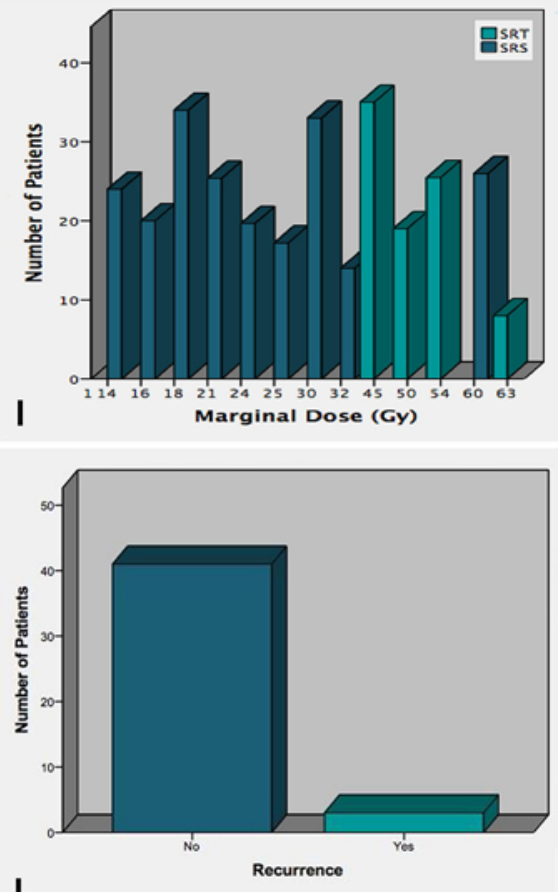

FIG. 1. Graphic representations of cohort characteristics: age at diagnosis (A); sex (B); presenting symptoms (C), including diplopia, headache (HA), dizziness (DZ), visual impairment (VI), trigeminal neuralgia, and orbital pain; side of the tumor (D); tumor volume (E); primary treatment $(\mathbf{F})$, which included surgery, SRS, and SRT; treatment modalities $(\mathbf{G})$ including endoscopic optic nerve decompression (EOND), endonasal transsphenoidal surgery (ENTS), frontotemporal craniotomy (FTC), transcranial orbitotomy (TCO), IMRT, EBRT, retrosigmoid approach (RS), transzygomatic approach (TZ), and CyberKnife (CK); completeness of resection after surgery $(\mathrm{H})$ including GTR and STR; marginal dose of SRS and SRT (I); number (n) of fractions of SRS and SRT (J); complications (K) including ophthalmoplegia, severe orbital pain, radiation-induced skin necrosis, and subacute infarct and homonymous hemianopia; and recurrence of tumor $(\mathrm{L})$. Figure is available in color online only. 
TABLE 2. Baseline visual function and outcome after treatment

\begin{tabular}{lcc}
\hline Visual Parameter & Initial & Final \\
\hline I VED & & \\
No VED & $9(20.5)$ & $21(47.7)$ \\
Mild VED & $20(45.5)$ & $10(22.7)$ \\
Moderate VED & $7(15.9)$ & $4(9.1)$ \\
Severe VED & $5(11.4)$ & $5(11.4)$ \\
NLP & $3(6.8)$ & $4(9.1)$ \\
C VED & & \\
No VED & $35(79.5)$ & $39(88.6)$ \\
Mild VED & $8(18.2)$ & $2(4.5)$ \\
Moderate VED & $0(0)$ & $2(4.5)$ \\
Severe VED & $1(2.3)$ & $1(2.3)$ \\
NLP & $0(0)$ & $0(0)$ \\
OD appearance & & \\
Normal & $22(50.0)$ & $28(63.6)$ \\
Pallor $(I)$ & $15(34.1)$ & $10(22.7)$ \\
Pallor $(I>C)$ & $6(13.6)$ & $4(9.1)$ \\
Pallor $(I<C)$ & $1(2.3)$ & $2(4.6)$ \\
IOP* & & $2(4.5)$ \\
I C C & $4(9.1)$ & $15(34.1)$ \\
I = C & $10(22.7)$ & $27(61.4)$ \\
I > C & $30(68.2)$ & $36(81.8)$ \\
EOM & $21(47.7)$ & $8(18.2)$ \\
Full & $23(52.3)$ & \\
Restricted & & \\
\hline
\end{tabular}

$\mathrm{C}=$ contralateral; $\mathrm{I}=$ ipsilateral.

* Normal $=12-22 \mathrm{~mm} \mathrm{Hg}$. intact, fully recovered, remained stable, and worsened, respectively, in $0 \%, 50 \%, 50 \%$, and $0 \%$ of the patients after STR alone; in $36.8 \%, 47.4 \%, 15.8 \%$, and $0 \%$ of the patients after STR with radiation treatment; in $57.1 \%, 0 \%, 28.6 \%$, and $14.3 \%$ of the patients after GTR alone; and in $56.2 \%$, $37.5 \%, 6.3 \%$, and $0 \%$ of the patients after radiation treatment (SRS or SRT) alone.

In the univariable regression analysis, sex and initial restricted EOM were significant independent factors associated with final EOM. Multivariable regression analysis was performed with adjustment for age, tumor volume, and primary treatment modality, which revealed that initial restricted EOM (OR 20.6, 95\% CI 18.7-77.0, p = 0.02) was a significant independent predictor of final limited EOM.

\section{Subgroup Analysis}

There was no statistically significant difference in terms of final VED $(p=0.15)$ and final EOM $(p=0.63)$ between surgery and radiation therapy (SRS or SRT), as shown in Table 4. There was also no statistically significant difference between open surgery and endoscopic surgery in terms of posttreatment VED $(\mathrm{p}=0.76)$ and EOM $(\mathrm{p}=0.34)$.

Among the patients who had undergone surgery, 8 (29.6\%) experienced improvement in EOM after STR, compared with none of the patients after GTR $(p=0.03)$. However, there was no statistically significant difference in terms of postoperative VED after GTR versus STR ( $p$ $=0.59$ ). Furthermore, there was no statistically significant

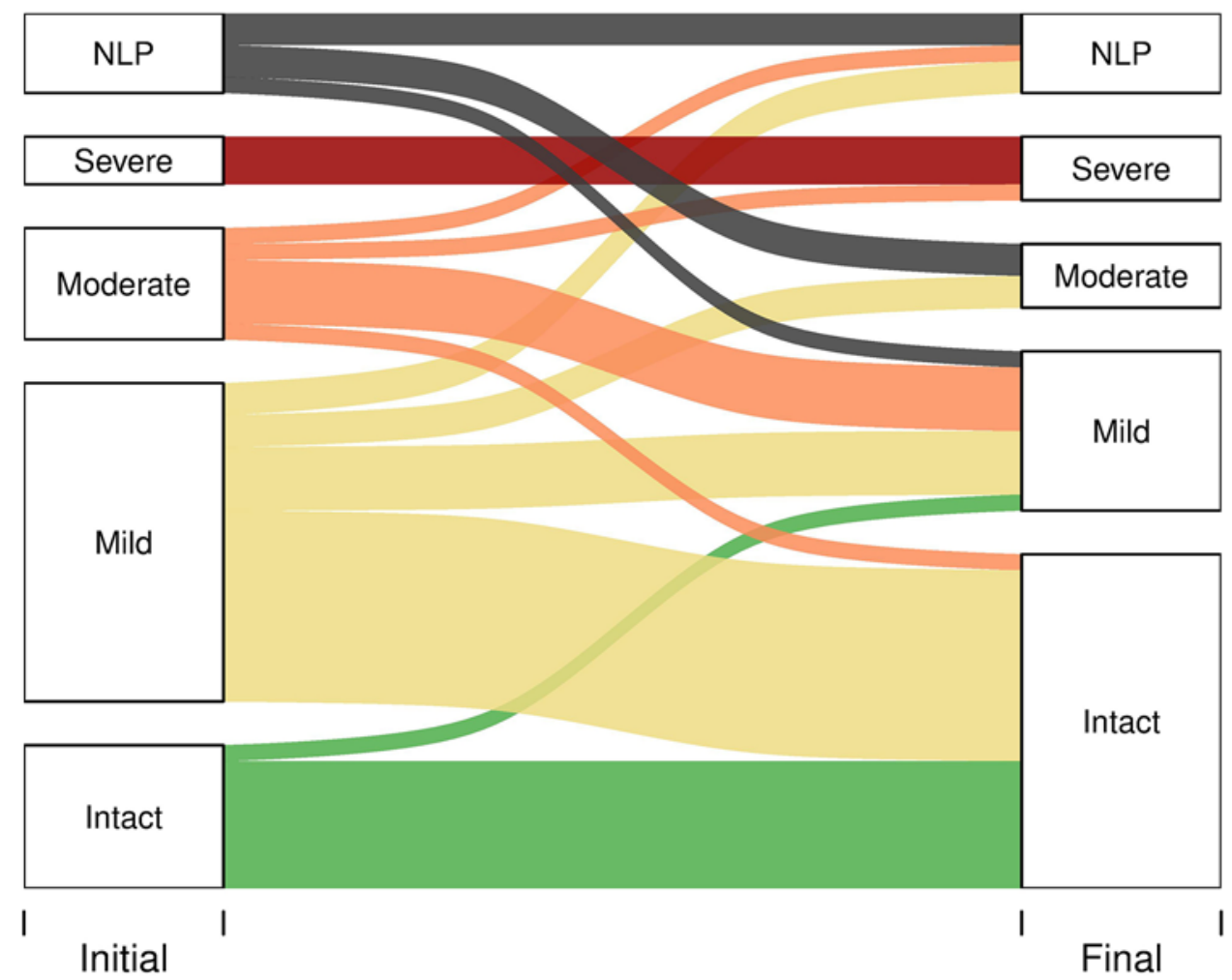

FIG. 2. Graphic representation (Sankey diagram) of VED in the ipsilateral eye before and after treatment. The width of the flowchart is proportional to the number of patients in each category. Figure is available in color online only. 


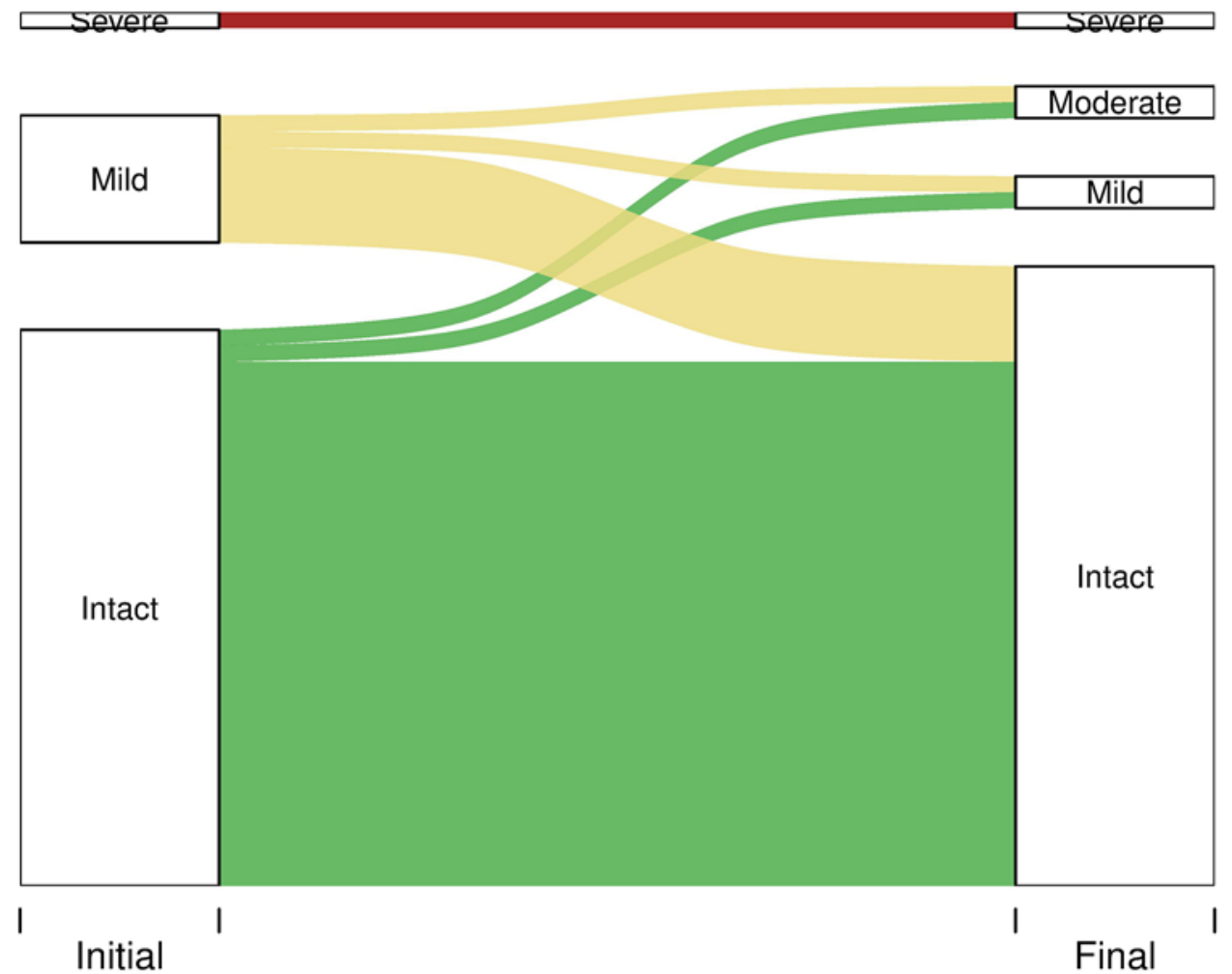

FIG. 3. Graphic representation (Sankey diagram) of VED in the contralateral eye before and after treatment. The width of the flowchart is proportional to the number of patients in each category. Figure is available in color online only.

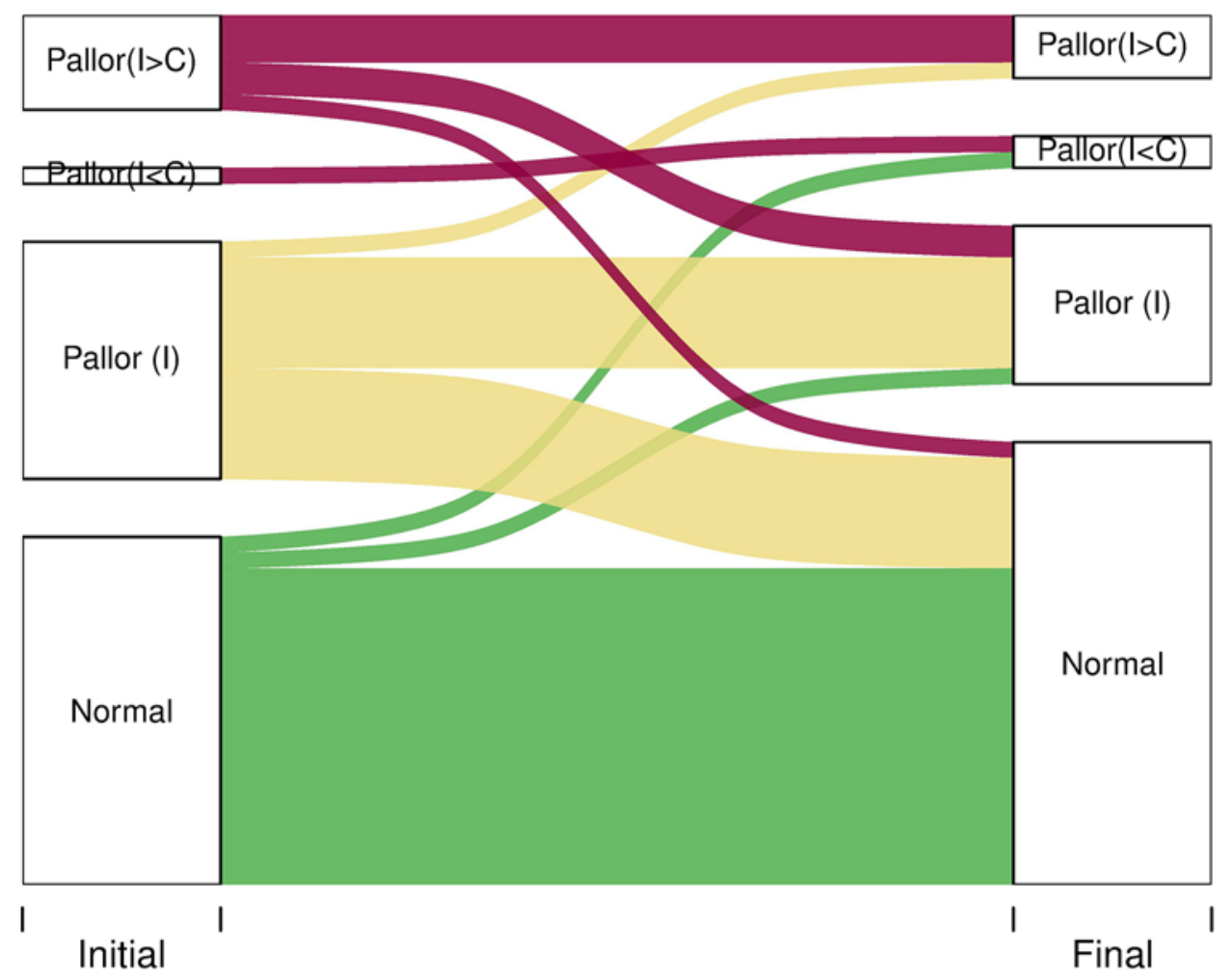

FIG. 4. Graphic representation (Sankey diagram) of OD appearance before and after treatment. The width of the flowchart is proportional to the number of patients in each category. $\mathrm{C}=$ contralateral; $\mathrm{I}=$ ipsilateral. Figure is available in color online only. 
Fatima et al.

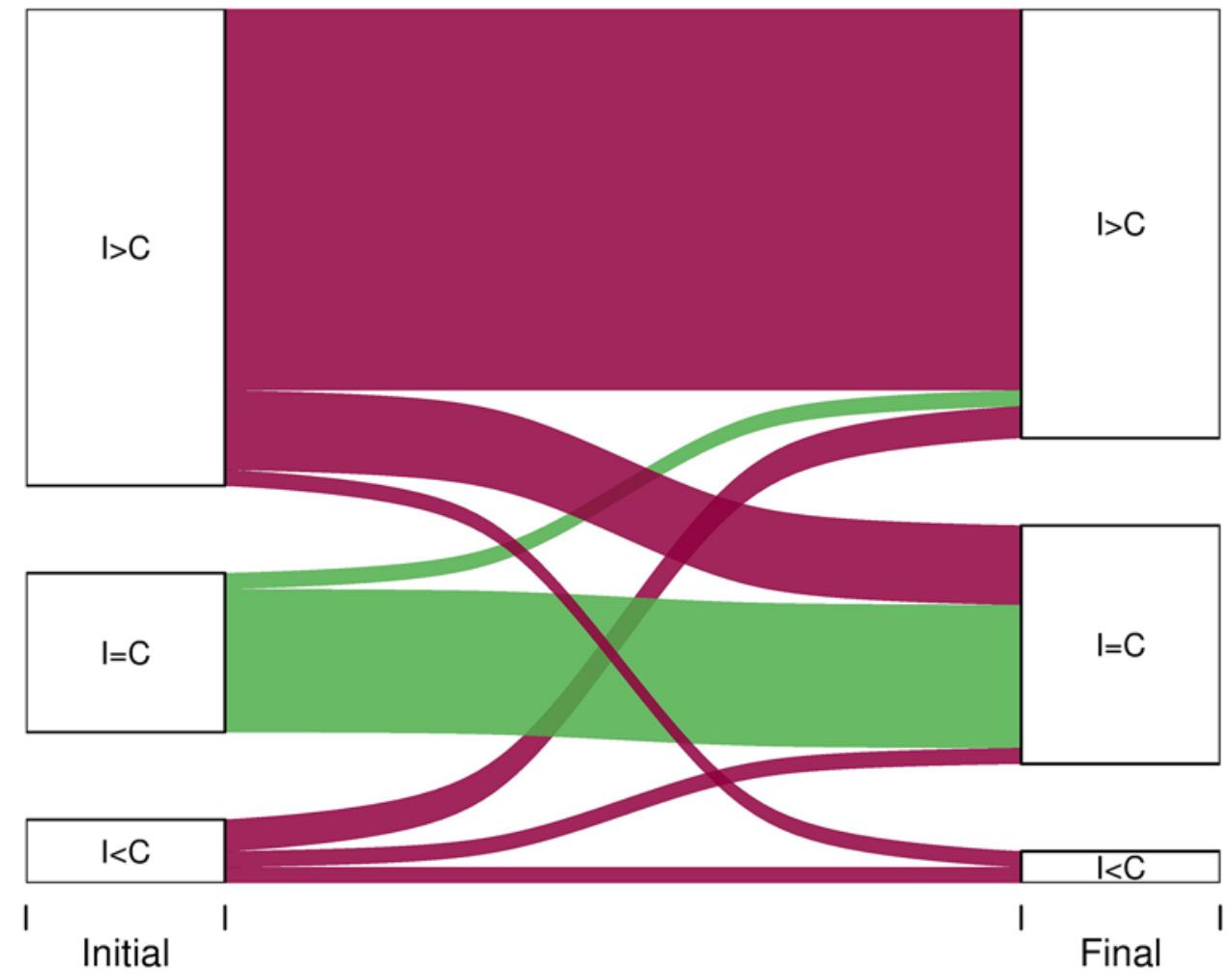

FIG. 5. Graphical representation (Sankey diagram) of IOP before and after treatment. The width of the flowchart is proportional to the number of patients in each category. Figure is available in color online only.

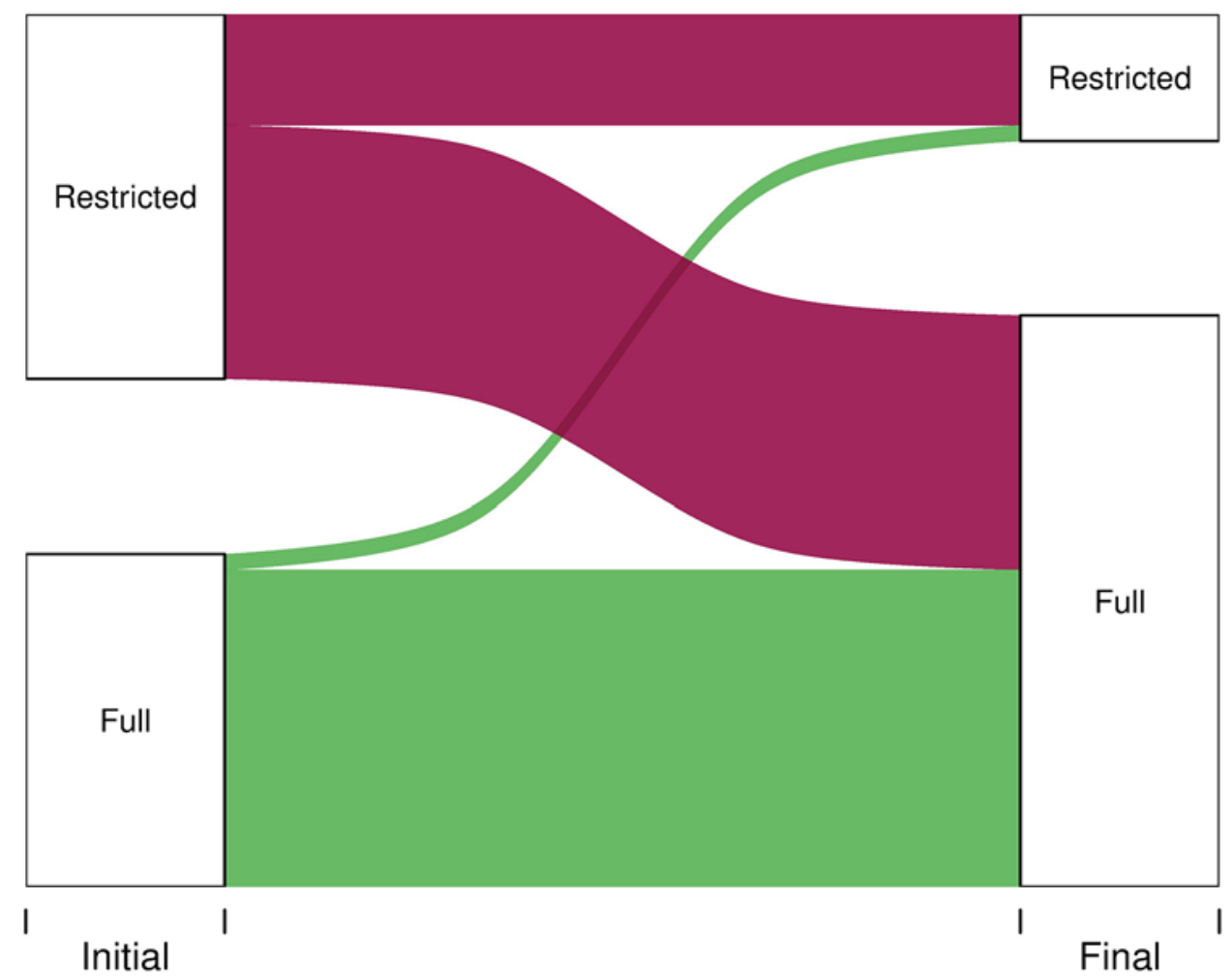

FIG. 6. Graphical representation (Sankey diagram) of EOM before and after treatment. The width of the flowchart is proportional to the number of patients in each category. Figure is available in color online only. 


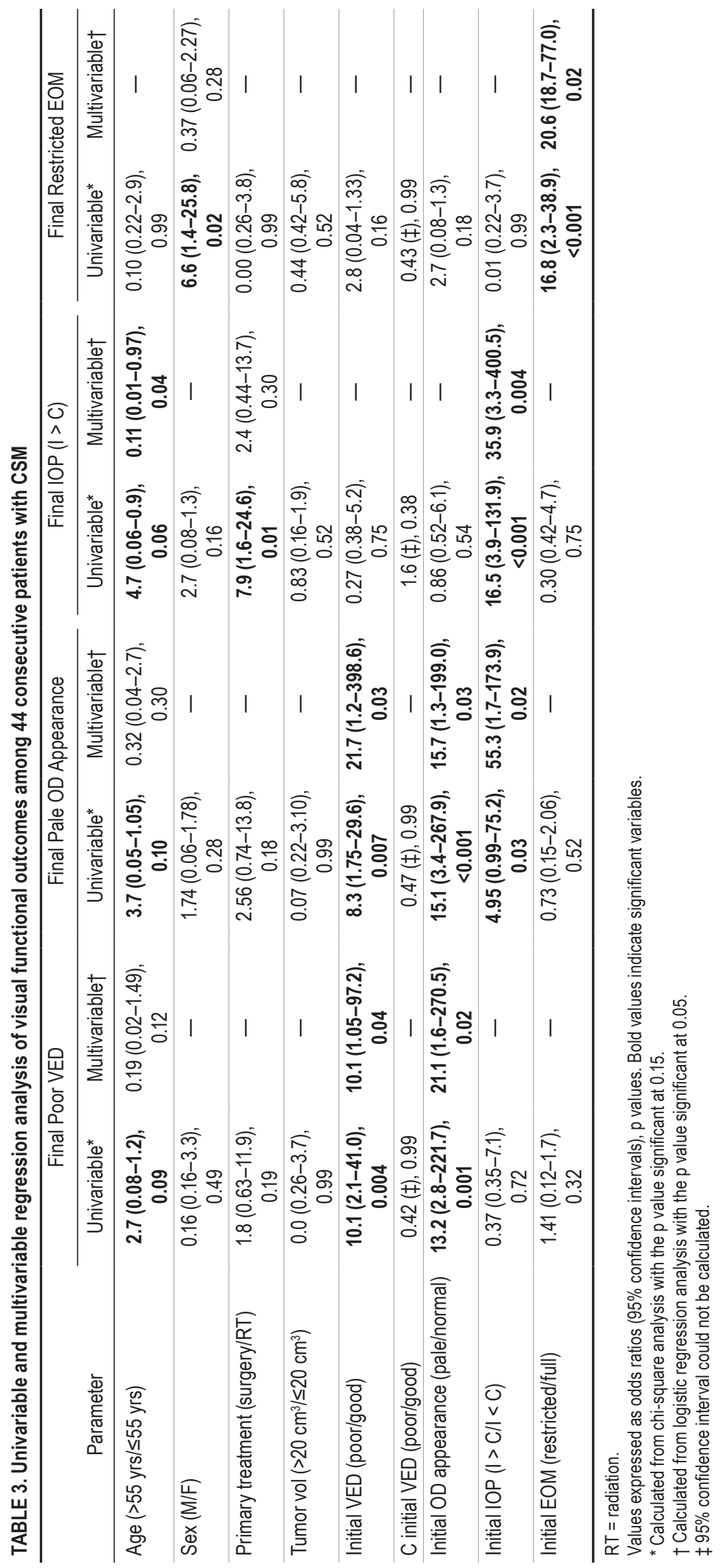


TABLE 4. Subgroup analysis based on the treatment modality impact on postoperative VED and EOM

\begin{tabular}{|c|c|c|c|c|c|c|}
\hline \multirow[b]{2}{*}{ Parameter } & \multicolumn{2}{|l|}{ Final VED } & \multirow[b]{2}{*}{ p Value } & \multicolumn{2}{|l|}{ Final EOM } & \multirow[b]{2}{*}{$\mathrm{p}$ Value } \\
\hline & Stable or Improvement & Worsened & & Stable or Improvement & Worsened & \\
\hline \multicolumn{7}{|l|}{ Treatment modality } \\
\hline Surgery & $21(77.8)$ & $6(22.2)$ & \multirow{2}{*}{0.15} & $8(88.9)$ & $1(11.1)$ & \multirow{2}{*}{0.63} \\
\hline RT & $16(94.1)$ & $1(5.9)$ & & $5(100.0)$ & $0(0.0)$ & \\
\hline \multicolumn{7}{|l|}{ Surgical approach } \\
\hline Open & $12(75.0)$ & $4(25.0)$ & \multirow{2}{*}{0.76} & $6(85.7)$ & $1(14.3)$ & \multirow{2}{*}{0.34} \\
\hline Endoscopic & $1(100.0)$ & $0(0.0)$ & & $2(100.0)$ & $0(0.0)$ & \\
\hline \multicolumn{7}{|c|}{ Completeness of resection } \\
\hline STR & $15(78.9)$ & $4(21.1)$ & \multirow{2}{*}{0.59} & $8(100.0)$ & $0(0.0)$ & \multirow{2}{*}{0.03} \\
\hline GTR & $6(75.0)$ & $2(25.0)$ & & $0(0.0)$ & $1(100.0)$ & \\
\hline \multicolumn{7}{|c|}{$\mathrm{RT}$ treatment modality } \\
\hline SRS & $12(100.0)$ & $0(0.0)$ & \multirow{2}{*}{0.20} & $5(100.0)$ & $0(0.0)$ & \multirow{2}{*}{0.07} \\
\hline SRT & $2(66.7)$ & $1(33.3)$ & & $0(0)$ & $0(0.0)$ & \\
\hline
\end{tabular}

difference between radiation techniques (SRS vs SRT) in terms of posttreatment VED $(p=0.20)$ and EOM $(p=$ 0.07).

\section{Discussion}

In this analysis of 44 patients with CSM, 35 patients (79.5\%) presented with VED in the ipsilateral eye, 20 of whom (45.5\%) improved; only 1 patient (2.8\%) had deterioration of their visual function posttreatment. A total of 27 patients (61.4\%) underwent resection of the CSM followed by either SRS or SRT, and only 17 patients (38.6\%) underwent primary SRS or SRT followed by surgery. All but 2 patients who underwent STR had radiation therapy as an adjuvant, whereas patients who underwent GTR did not require postoperative radiation therapy. In the multivariable analyses, the factors that independently predicted visual functional outcomes were initial ipsilateral VED assessment, initial ipsilateral OD appearance, initial IOP (ipsilateral compared to contralateral), and initial EOM. However, age, sex, primary treatment modality, tumor volume, and contralateral visual functions did not influence the outcome in patients with CSM, except for IOP (age, $p$ $=0.04)$.

We also performed a subgroup analysis to understand the impact of various treatment modalities in terms of improvement in postoperative visual functional outcomes. We found that patients who had undergone STR, compared to those who had undergone GTR, had either conservation or improvement in their postoperative EOM. Therefore, an understanding of these predictive parameters is of utmost importance in clinical decision-making and patient care.

We found that initial ipsilateral VED and OD appearance were predictive of posttreatment visual functional outcome in CSM, but contralateral VED was not. Although the most common clinical presentation of CSM is attributable to the involvement of cranial nerves (CNs) III, IV, V, and VI, ${ }^{16}$ the optic nerve (CN II) involvement remains a surrogate marker of a possible growing tumor. The extrinsic compression to the optic nerve against the bony structures of the skull base (clinoid process and optic canal) and the perioptic dura (i.e., falciform ligament), the encasement and compression of the internal carotid artery (ICA) and its branches, as well as the direct compression of the optic chiasm and optic tracts, are the pathophysiological basis for the visual compromise in patients with CSM. ${ }^{17,18}$ Specifically, encasement and stenosis of the cavernous segment of the ICA and disruption in the arterial blood flow to the small arterial branches in the parasellar or suprasellar regions due to mass effect are the most important mechanisms of visual impairment in patients with CSM..$^{19}$ This circulatory insufficiency leads to morphological alterations in the optic nerve in the form of loss of retinal ganglion cells and hence manifests as pale OD on fundoscopy. ${ }^{20}$

According to our results, the initial OD appearance was predictive of the posttreatment appearance of the OD in the ipsilateral eye $(\mathrm{p}=0.03)$. In our series, only 10 patients (22.7\%) had posttreatment recovery of their OD appearance. Indeed, this was attributable to the fact that almost all ischemic neuropathies are irreversible given the loss of healthy retinal ganglion fibers, except in the nonarteritic ischemic optic neuropathy in which the OD regains its appearance within 6 months of the events..$^{21}$ Furthermore, we found that older patients ( $>55$ years old) had a greater likelihood of a pale OD appearance posttreatment, although the relationship between the two factors was statistically nonsignificant $(p=0.10)$. However, this concept is contrary to the fact that older patients have a lower probability of regaining their visual status than younger individuals given the lower neuronal density in the older retina. ${ }^{22}$ Previous studies have suggested that men show more apparent changes in the retinal nerve fiber layer in their optic neuropathy eye than do women $(\mathrm{p}=0.003) .{ }^{23}$ However, we did not find a statistically significant difference in terms of OD appearance between the sexes $(\mathrm{p}=0.28)$.

The association of an elevation in IOP with corresponding underlying tumor is well known. ${ }^{6,23}$ In our study, posttreatment, 27 patients $(61.4 \%)$ had higher IOP in the ipsilateral than in the contralateral eye, 2 patients $(4.5 \%)$ had 
higher IOP in the contralateral than in the ipsilateral eye, and 15 patients $(34.1 \%$ ) had the same IOP in both eyes. For our analysis, we merged the patients with the same IOP in both eyes and the patients with lower IOP in the ipsilateral than the contralateral eye. We found that patients with higher ipsilateral IOP have a higher probability of elevated IOP in the ipsilateral eye posttreatment $(p=0.004)$. Moreover, we found that patients with initial restricted eye mobility had a greater likelihood of restricted eye mobility posttreatment $(\mathrm{p}=0.02)$. In our series, 15 patients $(34.1 \%)$ improved from restricted to full EOM; however, primary treatment modality or tumor volume did not affect the outcome of EOM.

In fact, the primary treatment modality in our study did not predict the visual functional outcomes in patients with CSM. Although there is no definite therapeutic algorithm for the management of CSM, radiosurgery with or without prior resection is considered the treatment of choice. The visual outcome in our study corresponds to the patient status at the last follow-up. In the case of patients who underwent a combination of treatments, such as surgery followed by SRS or SRT, intermediate measurements of visual function were not available. However, none of the patients who had undergone adjuvant SRS or SRT reported a subjective change in visual function during or immediately after SRS or SRT requiring an urgent ophthalmological evaluation. According to our analysis, 17/19 (89.4\%) patients who underwent STR had subsequent SRS or SRT, while none of the patients with GTR had adjuvant radiation treatment. Our results showed better visual functional outcomes among the patients who underwent STR followed by SRS or SRT as compared to those in patients who underwent GTR alone; therefore, we can extrapolate the positive impact of adjuvant SRS or SRT on the overall visual outcomes. However, further prospective studies are needed to ascertain the visual function changes after adjuvant SRS or SRT for CSM. Furthermore, a meta-analysis of 2000 patients with CSM revealed that patients who had undergone surgery followed by SRS had a higher rate of neurological morbidity than the patients who had undergone radiosurgery alone (59.6\% vs $25.7 \%) .{ }^{24}$ In addition, radiation therapy with a standard fractionation schedule (56 Gy/28 fractions) was found to result in a local tumor rate of $82 \%-95 \%$ at 5 years. ${ }^{25,26}$ In our study, various surgical approaches were adopted in 27 patients (61.4\%), while 12 patients (27.3\%) underwent primary SRS in the form of CyberKnife surgery and 5 patients (11.4\%) underwent SRT as a first-line treatment modality. However, we did not find any statistically significant differences in primary treatment modality in terms of the visual functional outcomes in patients with CSM.

Our study has several limitations. It is retrospective in design and includes a small patient cohort treated at a single institution, thereby limiting generalizability of the results. Moreover, the role, indications, and results of surgery are not reproducible across different series and should be considered at each patient level. In our series, as an alternative to classic approaches to the frontotemporal region for the resection of CSM, transcranial and endoscopic approaches to the orbit were needed to resect the orbital extension of the CSM, while the retrosigmoid approach was needed to resect a CSM with significant extension in the posterior fossa. Therefore, the heterogeneous location and extensions of the CSMs to the adjacent anatomical compartments of the skull base (orbit and posterior fossa) dictated the selection of the surgical approach, as well as the combination with other radiation techniques, making the results difficult to compare with those of other series.

Despite the limitations above, our study provides an important first look into the measures of pretreatment visual function that influence posttreatment visual outcome. Future prospective studies are needed to validate our regression models and guide clinical decision-making.

\section{Conclusions}

Our findings suggest that factors such as age, initial ipsilateral VED, initial ipsilateral OD appearance, initial IOP (ipsilateral compared to contralateral), and initial ipsilateral EOM are significant independent predictors of visual functional outcomes posttreatment in patients with CSM. However, sex, tumor volume, primary treatment modality, and contralateral visual function do not influence posttreatment visual function.

\section{References}

1. Couldwell WT, Fukushima T, Giannotta SL, Weiss MH. Petroclival meningiomas: surgical experience in 109 cases. $J$ Neurosurg. 1996;84(1):20-28.

2. DeMonte F, Smith HK, al-Mefty O. Outcome of aggressive removal of cavernous sinus meningiomas. J Neurosurg. 1994;81(2):245-251.

3. Kotapka MJ, Kalia KK, Martinez AJ, Sekhar LN. Infiltration of the carotid artery by cavernous sinus meningioma. J Neurosurg. 1994;81(2):252-255.

4. Levine ZT, Buchanan RI, Sekhar LN, et al. Proposed grading system to predict the extent of resection and outcomes for cranial base meningiomas. Neurosurgery. 1999;45(2):221230.

5. Sen C, Hague K. Meningiomas involving the cavernous sinus: histological factors affecting the degree of resection. $J$ Neurosurg. 1997;87(4):535-543.

6. Cusimano MD, Sekhar LN, Sen CN, et al. The results of surgery for benign tumors of the cavernous sinus. Neurosurgery. 1995;37(1):1-10.

7. O'Sullivan MG, van Loveren HR, Tew JM Jr. The surgical resectability of meningiomas of the cavernous sinus. Neurosurgery. 1997;40(2):238-247.

8. Liu JK, Forman S, Hershewe GL, et al. Optic nerve sheath meningiomas: visual improvement after stereotactic radiotherapy. Neurosurgery. 2002;50(5):950-957.

9. Spiegelmann R, Nissim O, Menhel J, et al. Linear accelerator radiosurgery for meningiomas in and around the cavernous sinus. Neurosurgery. 2002;51:1373-1380.

10. Margalit NS, Lesser JB, Moche J, Sen C. Meningiomas involving the optic nerve: technical aspects and outcomes for a series of 50 patients. Neurosurgery. 2003;53(3):523-533.

11. Wright JE, McNab AA, McDonald WI. Primary optic nerve sheath meningioma. Br J Ophthalmol. 1989;73(12):960-966.

12. Milker-Zabel S, Huber P, Schlegel W, et al. Fractionated stereotactic radiation therapy in the management of primary optic nerve sheath meningiomas. J Neurooncol. 2009;94(3):419-424.

13. Soldà $\mathrm{F}$, Wharram B, Gunapala R, Brada M. Fractionated stereotactic conformal radiotherapy for optic nerve sheath meningiomas. Clin Oncol (R Coll Radiol). 2012;24(8):e106e112. 
14. Jacob M, Wydh E, Vighetto A, Sindou M. Visual outcome after surgery for cavernous sinus meningioma. Acta Neurochir(Wien). 2008;150(5):421-429.

15. Koestler FA. The Unseen Minority: A Social History of Blindness in the United States. David McKay Co; 1976.

16. Cândido DNC, de Oliveira JG, Borba LAB. Microsurgical resection of cavernous sinus meningioma: 2-dimensional operative video. Oper Neurosurg (Hagerstown). 2018;15(6):E91.

17. Lao YX, Gao H, Xu JM. Vascular architecture of the human optic chiasm and bitemporal field defects. Chin Med J (Engl). 1990;103(5):406-414.

18. McIlwaine GG, Carrim ZI, Lueck CJ, Chrisp TM. A mechanical theory to account for bitemporal hemianopia from chiasmal compression. J Neuroophthalmol. 2005;25(1):4043.

19. Park W, Park JC, Han K, et al. Anterior optic pathway compression due to internal carotid artery aneurysms: neurosurgical management and outcomes. J Stroke. 2015;17(3):344353.

20. Patel HR, Margo CE. Pathology of ischemic optic neuropathy. Arch Pathol Lab Med. 2017;141(1):162-166.

21. Ho SF, Dhar-Munshi S. Nonarteritic anterior ischaemic optic neuropathy. Curr Opin Ophthalmol. 2008;19(6):461-467.

22. Harman A, Abrahams B, Moore S, Hoskins R. Neuronal density in the human retinal ganglion cell layer from 16-77 years. Anat Rec. 2000;260(2):124-131.

23. Costello F, Hodge W, Pan YI, et al. Sex-specific differences in retinal nerve fiber layer thinning after acute optic neuritis. Neurology. 2012;79(18):1866-1872.

24. Sughrue ME, Rutkowski MJ, Aranda D, et al. Factors affecting outcome following treatment of patients with cavernous sinus meningiomas. J Neurosurg. 2010;113(5):1087-1092.

25. Metellus P, Regis J, Muracciole X, et al. Evaluation of fractionated radiotherapy and gamma knife radiosurgery in cavernous sinus meningiomas: treatment strategy. Neurosurgery. 2005;57(5):873-886.
26. Shrieve DC, Hazard L, Boucher K, Jensen RL. Dose fractionation in stereotactic radiotherapy for parasellar meningiomas: radiobiological considerations of efficacy and optic nerve tolerance. J Neurosurg. 2004;101(suppl 3):390-395.

\section{Disclosures}

The authors report no conflict of interest concerning the materials or methods used in this study or the findings specified in this paper.

\section{Author Contributions}

Acquisition of data: Fatima. Analysis and interpretation of data: Fatima, Meola. Drafting the article: Fatima, Meola. Critically revising the article: Meola, Fatima, Ding, Han. Reviewed submitted version of manuscript: Fatima, Meola. Statistical analysis: Fatima, Ding, Han. Administrative/technical/material support: Fatima. Study supervision: Meola, Chang.

\section{Supplemental Information}

Online-Only Content

Supplemental material is available with the online version of the article.

Supplemental Tables 1 and 2. https://thejns.org/doi/suppl/ 10.3171/2020.2.JNS193009.

\section{Correspondence}

Antonio Meola: Stanford University School of Medicine, Stanford,CA.ameola@stanford.edu. 